\title{
Force-induced unfolding of human telomeric G-quadruplex: A steered molecular dynamics simulation study
}

\author{
Hui $\mathrm{Li}^{\mathrm{a}, \mathrm{b}}$, En-hua Cao ${ }^{\mathrm{b}}$, Thomas Gisler ${ }^{\mathrm{a}, *}$ \\ ${ }^{a}$ Universität Konstanz, Fachbereich Physik, 78457 Konstanz, Germany \\ ${ }^{\mathrm{b}}$ Institute of Biophysics. Chinese Academy of Sciences, Beijing 100101. China
}

\section{A R T I C L E I N F O}

\section{Keywords}

G-quadruplex

Unfolding

Steered molecular dynamics simulation

Single-molecule force spectrum

Intermediate state

\begin{abstract}
A B S T R A C T
We study the unfolding of a parallel G-quadruplex from human telomeric DNA by mechanical stretching using steered molecular dynamics (MD) simulation. We find that the force curves and unfolding processes strongly depend on the pulling sites. With pulling sites located on the sugar-phosphate backbone, the force-extension curve shows a single peak and the unfolding proceeds sequentially. Pulling sites located on the terminal nucleobases lead to a force-extension curve with two peaks and the unfolding is more cooperative. Simulations of the refolding of partially unfolded quadruplexes show very different behavior for the two different pulling modalities. In particular, starting from an unfolded state prepared by nucleobase pulling leads to a long-lived intermediate state whose existence is also corroborated by the free energy profile computed with the Jarzynski equation. Based on this observation, we propose a novel folding pathway for parallel G-quadruplexes with the human telomere sequence.
\end{abstract}

Although known for 40 years, G-quadruplex DNA structures formed by the self-assembly of guanine-rich poly- and oligonucleotides [1] have recently received renewed interest not only due to their importance for maintaining the very ends of chromosomes, the telomere [2], but also as possible targets for cancer therapy [3]. G-quadruplexes result from the hydrophobic stacking of several G-quartets, which are planes of four guanines held together by eight hydrogen bonds. The G-quartet stacking is further stabilized by cations (typically $\mathrm{K}^{+}$or $\mathrm{Na}^{+}$) located between two quartets which reduces the repulsion between the aromatic oxygen atoms in neighboring guanines $[4,5]$.

G-quadruplexes are comparable in stability with DNA duplexes [4]. The kinetics of their folding and unfolding has been studied with temperature-dependent circular dichroism and differential scanning calorimetry $[6-8]$ as well as with molecular dynamics (MD) simulation [9-13]. The detailed folding and unfolding pathways of $G$-quadruplexes remain elusive since they have defied study by single-molecule force experiments; equilibrium MD simulation, on the other hand, cannot characterize these processes due to their exceptionally slow kinetics. Stefl et al. studied the folding of a four-stranded intermolecular G-quadruplex of d(GGGG) by probing possible supramolecular intermediates in the formation pathway [11]. The folding of intramolecular quadruplexes containing the human telomeric DNA sequence -[TTAGGG]- is likely to be

\footnotetext{
* Corresponding author. Fax: +497531 883090.

E-mail address: thomas.gisler@uni-konstanz.de (T. Gisler)
}

more complicated because it involves the movement of the loop region (-[TTA]-).

The difficulties for studying G-quadruplex unfolding by equilibrium molecular simulation can be overcome by steered MD simulation [14,15]: the ends of the molecule are linked to Hookean springs serving as force sensors whose ends move in opposite directions with constant velocity. This method is able to give the non-equilibrium force $f(l)$ as a function of the DNA end-to-end distance $l$, similar to single-molecule force experiments [16]. Free energy profiles which allow to identify intermediate states can be obtained from non-equilibrium force-extension curves using the Jarzynski equation $[17,18]$.

In the present publication, steered MD simulation was used to study the unfolding of the parallel G-quadruplex from human telomeric DNA. By attaching the Hookean springs to different sites within the molecule (namely, to the terminal sugar backbone or to terminal bases), different force-extension curves are obtained which show one or two main peaks. The differences in the force curves are caused by different unfolding pathways, either characterized by a sliding mode or by a metastable intermediate state. The calculation of the free energy profile and refolding simulations corroborates the presence of the intermediate state in the case of pulling on the terminal nucleobases.

\section{Methods}

All simulations were carried out using the MD software package GROMACS 3.3 [19] with the AMBER 03 force field [20] and the 
Tip3P water model [21]. Simulations were run in the NPT ensemble. The temperature was kept constant at a temperature $T=300 \mathrm{~K}$ by coupling to a Berendsen thermostat. The pressure was kept constant at $p=1$ bar using anisotropic coupling to a Berendsen barostat [22]. All bonds were constrained using the LINCS algorithm [23]. An integration time step of $2 \mathrm{fs}$ was used. Lennard-Jones interactions were calculated using a cutoff of $1 \mathrm{~nm}$. At a distance smaller than $0.9 \mathrm{~nm}$, electrostatic interactions were calculated explicitly, whereas long-range electrostatic interactions were calculated by particle-mesh Ewald summation [24].

The 22-mer G-quadruplex of the human telomeric DNA sequence $5^{\prime}-\mathrm{AG}_{3}[\mathrm{TTAGGG}]_{3}-3^{\prime}$ was studied. The initial atom coordinates were taken from the crystal structure of the parallel quadruplex (PDB entry 1KF1 [25]; see Supporting Materials, Fig. S1). The quadruplex was solvated in a cubic box of $6.5 \times 6.5 \times 10 \mathrm{~nm}^{3} ; 22 \mathrm{~K}^{+}$ions were added to provide electroneutrality, three of which were positioned in the center of the quadruplex channel. An energy minimization of 1000 steps using the steepest descent algorithm was followed by a 400 ps position-constrained MD simulation in order to equilibrate water and ions. A subsequent MD simulation of 1 ns was performed to equilibrate the entire system.

For the steered MD simulations, two Hookean springs with same force constant $\left(k_{0}=1000 \mathrm{~kJ} /\left(\mathrm{mol} \mathrm{nm}^{2}\right)\right)$ were attached at the ends of the quadruplex in different ways: (i) to stretch from the sugar backbone, two springs were attached to $\mathrm{O5}^{\prime}$ atom of $\mathrm{A} 1$ and to the $03^{\prime}$ atom of $\mathrm{G} 22$, respectively; (ii) to stretch from the nitrogen atom of the terminal base, the two springs were attached to the N6 atom of $A 1$ and to the N2 atom of G22, respectively.

Force was applied by moving the two springs in opposite directions with constant velocity $v$ (see Supporting Materials, Fig. S1),

$Z_{i}(t)=Z_{i}(0) \pm v t$

where $v=1 \mathrm{~nm} / \mathrm{ns}$ is the constant pulling velocity, $z_{i}(0)$ is the initial position of the DNA end, $Z_{i}(t)$ is the position of the clamped end of the spring at time $t$ and $i$ refers to the $5^{\prime}$ or $3^{\prime}$ ends. Forces at the two ends were recorded at every time step.

$f_{i}(t)=k_{0}\left[z_{i}(t)-Z_{i}(t)\right]$

The force curves were smoothed with an FFT filter.

Free energy changes during the stretching were calculated based on the Jarzynski equation which relates the free energy $G(l)$ with the work $W(l)$ performed to stretch the molecule to the end-to-end distance $l=\left|z_{5}^{\prime}-z_{3}^{\prime}\right|$ in non-equilibrium conditions [17].

$\exp \left(-G(l) / k_{\mathrm{B}} T\right)=\overline{\exp \left(-W(I) / k_{\mathrm{B}} T\right)}$

where the overbar denotes the average over all possible trajectories. We created 50 pulling trajectories with initial conformations randomly selected from the equilibrium simulation. In order to save computer time, we decreased the simulation box to $5 \times 5 \times 6 \mathrm{~nm}^{3}$. The free energies using weighted histogram methods as ensemble average [26] were calculated using the expression developed by Hummer and Szabo [18].

\section{Results}

\section{Equilibrium simulations}

The stability of the G-quadruplex was verified by a 1 ns free MD simulation and comparison with the initial crystal structure. When three $\mathrm{K}^{+}$ions are positioned within the central channel of the Gquadruplex, one of them is readily expelled, leaving the remaining two cations located between the quartet planes. The resulting structure is stable, reflected by the small and constant overall root-mean-square displacement (RMSD) values $[(1.9 \pm 0.28) \mathrm{A}]$ after 200 ps (see Supporting Materials, Fig. S2). In contrast, when no ions are in the channel, the structure is unstable and the overall RMSD continuously increases during the simulation (Fig. S2). The importance of the $\mathrm{K}^{+}$ions for the stability of the $\mathrm{G}$-quadruplex structure is further underlined by the fact that the RMSD of the guanines after equilibration $[(0.98 \pm 0.16) \AA]$ is by about a factor of 2 smaller than the RMSD of the other bases $[(2.1 \pm 0.4) \AA]$ (Fig. S2). In the following, we focus on force-induced unfolding in the presence of $\mathrm{K}^{+}$ions.

\section{Force-extension curves and force-induced conformation changes}

Fig. $1(A)$ and (B) shows the DNA end-to-end distance $I(t)$ and the corresponding forces acting on the ends of the molecule as a function of simulation time. When the springs are connected to the backbone ( $\mathrm{O5}^{\prime}$ of $\mathrm{A} 1$ and $\mathrm{O}^{\prime}$ of $\mathrm{G} 22$, see inset in Fig. 1(A)), the force-extension curve shows a single peak at about $750 \mathrm{ps}$ (Fig. $1(A)$ ). When the springs are connected to the terminal nucleobases (N6 of A1 and N2 of G22, see inset in Fig. 1(B)), the forceextension curve shows two distinct peaks at about $600 \mathrm{ps}$ and 1100 ps which are separated by a trough at about $750 \mathrm{ps}$ (Fig. 1(B)). The large difference between the force curves in Fig. $1(A)$ and (B) indicates that the pulling at different sites results in force-induced unfolding of the G-quadruplex along different pathways.

The difference in the unfolding pathways is further revealed by the distance of the guanine bases to the molecular center during stretching, as shown in Fig. 1(C) and (D), as well as by the number of hydrogen bonds (see Supporting Materials, Fig. S3). During backbone pulling, all the guanine bases start to deviate sequentially from their equilibrium positions after about $700 \mathrm{ps}$ (Fig. 1(C)). This onset coincides with the peak in the force curves in Fig. 3(A). At the same time, the number of hydrogen bonds begins to decrease markedly (Fig. S3(a)), which implies that the G-quadruplex structure starts to break up. Although the distances of the guanine bases to the molecular center continue to increase after $1200 \mathrm{ps}$, the number of hydrogen bonds becomes stable of about 10 , reflecting that some local structure remains. In contrast, during nucleobase pulling only G22 starts to deviate from its original position at about $600 \mathrm{ps}$, followed by G2 at 1100 ps, while the other bases deviate substantially from their equilibrium position only after $1300 \mathrm{ps}$ (Fig. 1(D)). This indicates that the unfolding of the G-quadruplex is triggered by the cooperative dissociation of guanine bases from their quartet planes. The dissociation of G22 and G2 coincides with the two peaks (at $600 \mathrm{ps}$ and $1100 \mathrm{ps)}$ ) in the force-extension curve shown in Fig. 1(B). The number of hydrogen bonds rapidly drops from 24 to 18 at about $600 \mathrm{ps}$, and remains at about 16 until 1200 ps (Fig. S3(b)). The first loss of six hydrogen bonds is related to the detachment of $\mathrm{G} 22$ from its quartet plane, while the plateau in the hydrogen bond number for $700 \mathrm{ps} \leqslant t \leqslant 1200 \mathrm{ps}$ indicates the formation of a relatively stable, intermediate state.

More detailed structural changes along the trajectory are illustrated in Fig. 2 and Supporting Materials Movie S1 and S2, in which the different unfolding pathways are clearly visible. For both pulling modalities, only A1 is significantly extended at the beginning of stretching ( $t \leqslant 500 \mathrm{ps}$ ). Later on, the unfolding follows different directions for the different pulling modalities. For backbone pulling the relative positions of $(\mathrm{G} 14, \mathrm{G} 15$, and $\mathrm{G} 16)$ to $(\mathrm{G} 20, \mathrm{G} 21$, and $\mathrm{G} 22$ ) are almost unchanged, as well as the relative positions of (G8, G9, and G10) to (G2, G3, and G4), forming two local structures (Fig. 2(A)). When the molecule is stretched to an end-to-end distance of $8 \mathrm{~nm}$ at the end of the simulation $(2000 \mathrm{ps})$, one of the local structures is still present (Fig. 2(B)).

For nucleobase pulling, continuous stretching induces a complete detachment of G22 from its quartet plane, rotating perpen- 

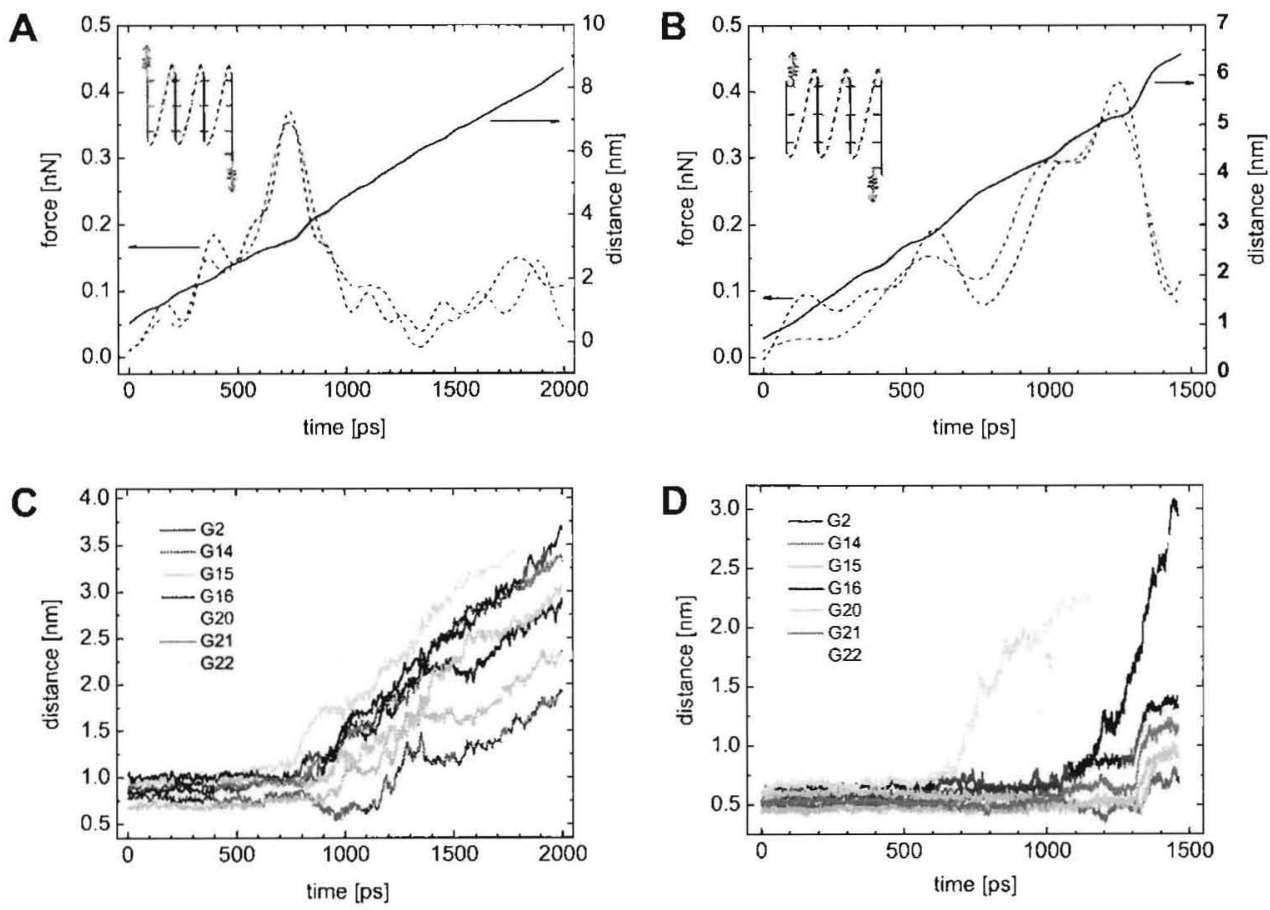

Fig. 1. End-to-end distance $l(t)$ (black solid lines) and forces exerted at the $5^{\prime}$ and $3^{\prime}$ ends of the G-quadruplex (red and blue dashed lines, respectively) as a function of simulation time $t$. Data shown are from a single MD run during $(A)$ backbone pulling and $(B)$ during nucleobase pulling. Distance of guanines to the molecular center during stretching (C) for backbone pulling and (D) for nucleobase pulling. For the sake of clarity, traces bf G3, G4, G8, G9, and G10 are omitted in (C) and (D), but show similar behavior to the ones of G14 to G21.
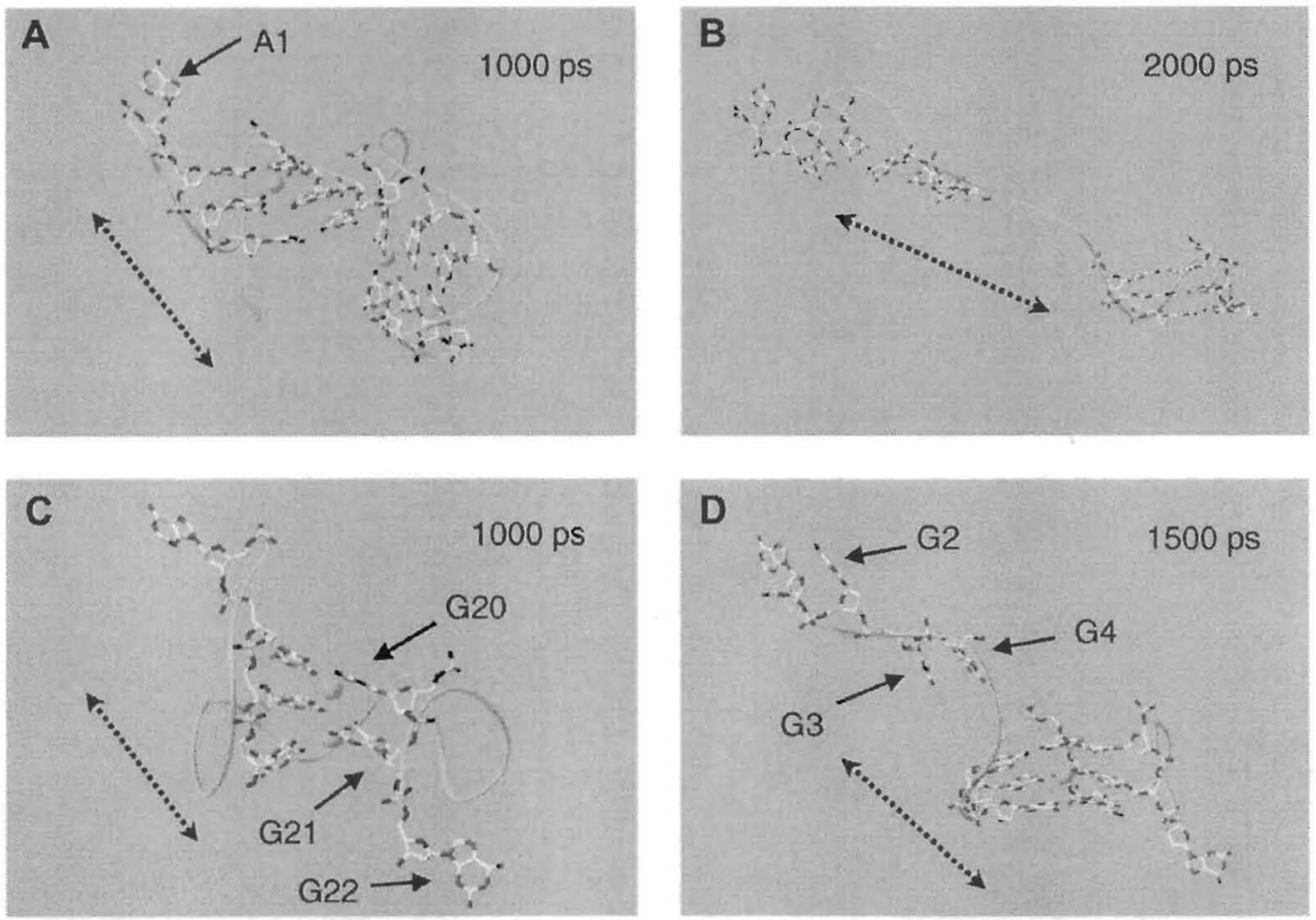

Fig. 2. Evolution of the structure of the $G$-quadruplex during backbone pulling $(A, B)$ and nucleobase pulling $(C, D)$. (A) After $t=1000$ ps during backbone pulling, the three quartet planes are separated into two parts. (B) $t=2000 \mathrm{ps}$ : a remnant local structure is formed by the six guanines in the lower right. (C) After $t=1000$ ps during nucleobase pulling, G22 breaks off the quartet plane while the other 11 guanines remain almost unchanged. (D) At the end of the simulation ( $t=1500 \mathrm{ps)}$ of nucleobase pulling, G3 and G4 break away from their quartet plane. Blue lines represent the sugar-phosphate backbone. For better visibility the elongated structures were rotated; the dashed arrows mark the pulling direction. 

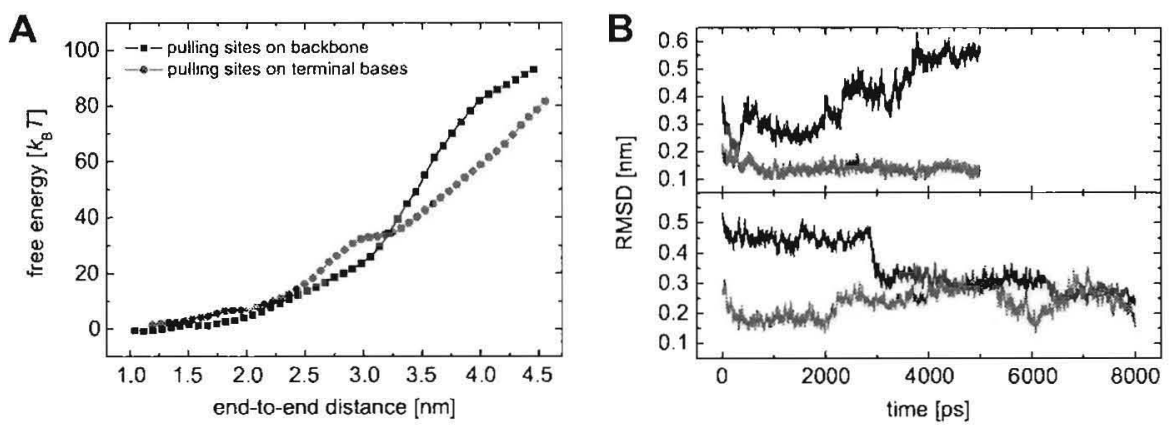

Fig. 3. (A) Free energy change $G(l)$ as a function of the end-to-end distance I for backbone and nucleobase pulling. respectively, calculated with Eq. (1.3) from 50 pulling trajectories. (B) Root-mean-square displacement (RMSD) of guanines with respect to the crystal structure as a function of time during free MD simulation. with starting structures taken from the stretching trajectory obtained by steered MD simulation. Upper panel: pulling sites located on the backbone; starting with partially unfolded structures after $750 \mathrm{ps}$ (red line) and after $800 \mathrm{ps}$ (black line); lower panel: pulling sites located on the terminal nucleobases; starting with structures after 600 ps (red line) and $1000 \mathrm{ps}$ (black line).

dicularly to the quartet plane while the other bases remain at their positions (Fig. 2(C)); at about the same time, the first peak in the force curves appears (Fig. 1(B)). This intermediate structure remains intact in the following 300 ps despite the increasing extension. Later one $\mathrm{K}^{+}$is released into the solvent, and $\mathrm{G} 2$ starts to deviate strongly from its equilibrium position (see Movie S2), leading to the second force peak at $1200 \mathrm{ps}$. After that, the G-quadruplex deforms quickly. At the end of the simulation (1500 ps), G3 and G4 are dissociated from their quartet plane but a local structure formed by eight guanine bases is still preserved (Fig. 2(D)).

\section{Free energy profiles}

Fig. 3(A) shows the free energy $G(l)$ as a function of the end-to-end distance ( for both the backbone and the nucleobase pulling modality, calculated from 50 non-equilibrium trajectories with Eq. (1.3). The slight increase of the free energy at small end-to-end distances $l<2.3 \mathrm{~nm}$ reflects the extension of A11. For distances $2.3 \mathrm{~nm}<1<3.0 \mathrm{~nm}$, the free energy increases more rapidly for terminal nucleobase than for backbone pulling. Pulling at the terminal nucleobases results in a shoulder of about $35 k_{\mathrm{B}} T$ at a distance of $3.3 \mathrm{~nm}$ which corresponds to the trough in the force-extension curve shown in Fig. 1(B). The free energy for backbone pulling, on the other hand, shows a more gradual increase with end-to-end distance.

\section{Refolding}

We attempted to simulate refolding of stretched G-quadruplexes by free MD simulation using structures from the stretching trajectory as starting data. For the backbone pulling modality, the molecule is found to either fold back to its equilibrium structure or to unfold entirely, depending on the amount the molecule had been stretched. Fig. 3(B) shows the RMSD of the guanines with respect to the equilibrium structure during free MD simulation. When the starting structure is taken from the coordinates at 750 ps of the pulling trajectory (Fig. 1(A)), the RMSD reaches a small value of about $0.15 \mathrm{~nm}$ after about $1 \mathrm{~ns}$ and remains constant thereafter (Fig. 3(B) upper panel, red line), which shows that the stretched structure at $750 \mathrm{ps}$ folds back to its equilibrium structure after releasing the force. In contrast, if the starting structure is taken from the same stretching trajectory at $800 \mathrm{ps}$, the molecule is observed to unfold entirely after the $5 \mathrm{~ns}$ free MD simulation, as indicated by the continuous increase of the RMSD (Fig. 3(B) upper panel, black line).

In the nucleobase pulling modality, the molecule neither folds back to its initial conformation nor totally unfolds within our simulation time. Fig. 3(B), lower panel, shows the RMSD of the gua- nines with respect to the equilibrium structure with the starting structures taken from coordinates at $600 \mathrm{ps}$ and $1000 \mathrm{ps}$ of the stretching trajectory. After about 3 ns, both RMSD values reach about $0.3 \mathrm{~nm}$ and remain nearly constant, which suggests that the two starting structures fold to a similar state which is, however, different from the equilibrium structure. Initial and final structure of one refolding process is shown in Supporting Materials Fig. S4. In $8 \mathrm{~ns}$, the molecule folds to a conformation in which G20 and G21 take the position of G21 and G22 in the crystal structure, forming two quartet planes with G3, G9, G15, G20 and G4, G10, $\mathrm{G} 16, \mathrm{G} 21$, respectively, which are stabilized by a $\mathrm{K}^{+}$ion.

\section{Discussion}

The different positions of the Hookean springs for nucleobase and backbone pulling lead to dramatically different force-extension curves which shows that the different pulling modes probe different unfolding pathways. Backbone pulling leads to a sequential loss of hydrogen bonding. while nucleobase pulling is characterized by an early, localized break-off of G22 and, later on, G2, from the quadruplex, followed by a rather cooperative unbinding at about $1300 \mathrm{ps}$. The wide plateau in the hydrogen bond number at about 15 for 700 ps $\leqslant t \leqslant 1200$ ps shown in Fig. S3(b) and the deep trough in the force-extension curve in Fig. 2(B) suggests that the pulling generates a relatively stable intermediate structure. The calculation of the free energy $G(I)$ shows a shoulder of about $35 k_{\mathrm{B}} T$ at an end-to-end distance of $3 \mathrm{~nm}$ which corresponds to the trough in the force-extension curve for nucleobase pulling. The absence of a clear local minimum in $G(l)$ at $3 \mathrm{~nm}$ which would give clear evidence for a thermodynamically metastable intermediate could be due to errors in the estimation of $G(I)$ induced by the relatively low number of non-equilibrium trajectories used for calculating the free energy. However, the fact that in the refolding simulation starting from the partially unfolded structure we find a long-lived intermediate structure with two complete and one partial quartet plane, different from the ground state, indicates that this intermediate indeed is a structure which is produced typically, and not just marginally, by nucleobase pulling. Assuming that the lifetime $\tau=4 \mathrm{~ns}$ observed in the refolding simulation is representative, we estimate an activation energy $E_{a}=k_{\mathrm{B}} T \ln (\tau \gamma)=$ $8 k_{\mathrm{B}} T$ required for a transition to the ground state by the Eyring equation for an overdamped system [27], assuming a momentum relaxation time $\gamma^{1}=1 \mathrm{ps}$ for a DNA duplex.

For backbone pulling, the free energy $G(l)$ is much smoother, which is in line with the continuous denaturation of the quadruplex induced by pulling, and the absence of a long-lived intermediate in the refolding of the moderately unfolded quadruplex. 


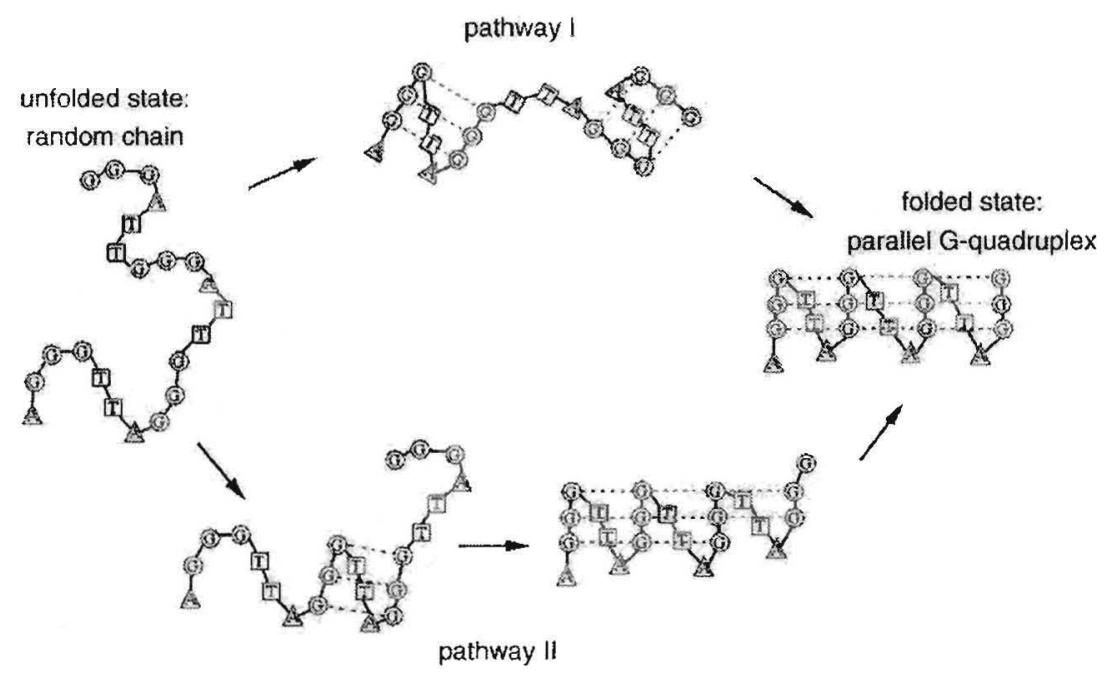

Fig. 4. Model for folding of the parallel G-quadruplex based on the structures observed in the unfolding and refolding simulations. Dashed lines represent Hoogsteen pairing between guanine bases.

How a G-quadruplex forms from a random chain is an open question and cannot be followed with the present MD simulations. A "nucleation-slippage" model was put forward by Stefl et al. for the formation of intermolecular G-wires [11]. The model by Mashimo and Sugiyama for the formation of a hybrid intramolecular G-quadruplex suggests that a hairpin structure is first formed, followed by a chair conformation and, finally, a strand flip within the chair yielding a hybrid G-quadruplex [28]. In our steered MD simulation, different unfolding pathways of the parallel G-quadruplex are observed depending on the anchoring sites. According to these observations, we propose two folding pathways for the parallel Gquadruplex, as shown in Fig. 4. In folding pathway I, the random chain first forms two hairpin structures at the two ends, foliowed by association of the two hairpins to the quadruplex. Trapping of two $\mathrm{K}^{+}$is most likely to occur in the second step. In folding pathway II, a hairpin is first formed in the center of the molecule, followed by the association of the other two arms. This step might proceed via slippage of one or two arms, similar to intermolecular quadruplex formation [11]. At least for the last stages of quadruplex folding. the long lifetime of the metastable intermediate observed in the nucleobase pulling simulations suggests that the fastest way of folding proceed's via pathway I which appears to be less prone to kinetic trapping, rather than via pathway II.

In principle our simulation data can be compared to force-extension curves obtained from single-molecule force experiments. However, the pulling velocities used here are, due to limited computer time, by several orders of magnitude larger than the ones used in current experiments [29]. This may result in a significant overestimation of unfolding forces. Nonetheless, this should not affect the existence of the intermediate state during the unfolding predicted by our simulations and the folding pathway we suggested. An attractive candidate for experimentally testing the folding pathway presented here is single-molecule fluorescence resonance energy transfer (FRET) which allows to continuously monitor the distance between terminal bases labeled with donor and acceptor fluorescence dyes during the folding process.

\section{Acknowledgments}

This work was supported by the Deutsche Forschungsgemeinschaft and grants (90206041 and 30570461) from the National Science Foundation of China.

\section{Appendix A. Supplementary data}

Supplementary data associated with this article can be found, in the online version, at doi:10.1016/j.bbrc.2008.12.006.

\section{Refererices}

[1] M. Gellert, M.N. Lipsett, D.R. Davies, Helix formation by guanylic acid. Proc Natl. Acad. Sci. USA 48 (1962) 2013-2018

(2) R.H. Shafer, I. Smimov, Biological aspects of DNA/RNA quadruplexes, Biopolymers 56 (2000) 209-227.

13] S. Neidle, M.A. Read, G-quadruplexes as therapeutic targets, Biopolymers 56 (2000) 195-208.

14] A.N. Lane, J.B. Chaires. R.D. Gray. J.O. Trent, Stability and kinetics of Gquadruplex structures, Nucleic Acids Res. 36 (2008) 5482-5515.

[5] J.L. Mergny. A. De Cian, A. Ghelab, B. Sacca, L. Lacroix, Kinetics of tetramolecular quadruplexes. Nucleic Acids Res. 33 (2005) 81-94.

16] B.I. Kankia, LA. Marky. Folding of the thrombin aptamer into a G-quadruplex with $\mathrm{Sr}^{2+}$ : stability, heat, and hydration, J. Am. Chem. Soc. 123 (2001) 1079910804.

[7] C.M. Olsen, W.H. Gmeiner, L.A. Marky, Unfolding of G-quadruplexes: energetic, and ion and water contributions of G-quartet stacking. J. Phys. Chem. B 110 (2006) 6962-6969.

[8] R.D. Gray. J.B. Chaires, Kinetics and mechanism of $\mathrm{K}^{+}$and $\mathrm{Na}^{+}$-induced folding of models of human telomeric DNA into G-quadruplex structures, Nucleic Acids Res. 36 (2008) 4191-4203.

19| E. Gavathiotis, M.S. Searle, Structure of the parallel-stranded DNA quadruplex d(TTAGGGT) 4 containing the human telomcric repeat: evidence for A-tetrad formation from NMR and molecular dynamics simulations. Org. Biomol. Chem. 1 (2003) 1650-1656.

[10] M. Cavallari, A. Calzolari, A. Garbesi, R. Di Felice, Stability and migration of metal ions in G4-wires by molecular dynamics simulations, I. Phys. Chem. B 110 (2006) 26337-26348

[11] R. Stefl, T.E. Cheatham, N. Spackova, E. Fadrna, I. Berger, J. Koca, J. Sponer, Formation pathways of a guanine-quadruplex DNA revealed by molecula: dynamics and thermodynamic analysis of the substates, Biophys. J. 85 (2003) 1787-1804.

[12] E. Fadrna, N. Spackova, R. Stefl. J. Koca. T.E. Cheatham, J. Sponer. Molecular dynamics simulations of guanine quadruplex loops: advances and force field limitations, Biophys. J. 87 (2004) 227-242

[13] M. Rueda, F.J. Luque, M. Orozco, G-quadruplexes can maintain their structure in the gas phase. J. Am. Chem. Soc. 128 (2006) 3608-3619.

[14] M. Sotomayor, K. Schulten. Single-molecule experiments in vitro and in silico. Science 316 (2007) 1144-1148.

[15] F. Grater, J.H. Shen, H.L. Jiang. M. Gautel, H. Grubmüller, Mechanically induced titin kinase activation studied by force-probe molecular dynamics simulations. Biophys. J. 88 (2005) 790-804.

(16] J. Zlatanova. K. van Holde. Single-molecule biology: what is it and how does it work? Mol Cell 24 (2006) 317-329.

117] C. Jarzynski, Nonequilibrium equality for free energy differences, Phys. Rev. Lett. 78 (1997) 2690-2693

[18] G. Hummer, A. Szabo, Free energy reconstruction from nonequilibrium singlemolccule pulling experiments, Proc. Natl. Acad. Sci. USA 98 (2001) 3658-3661. 
[19] D. Van der Spoel, E. Lindahl, B. Hess, G. Groenhof. A.E. Mark, H.J.C. Berendsen, GROMACS: fast, flexible, and free, J. Comp. Chem. 26 (2005) 1701-1718.

[20] E.J. Sorin, V.S. Pande, Exploring the helix-coil transition via all-atom equilibrium ensemble simulations, Biophys. J. 88 (2005) 2472-2493.

[21] M.W. Mahoney. L.W. Jorgensen, A five-site model for liquid water the reproduction of the density anomaly by rigid, nonpolarizable potential functions. J. Chem. Phys. 112 (2000) 8910-8922

(22) H.J.C. Berendsen, J.P.M. Postma, W.F. van Gunsteren, A.D. Nola, J.R. Haak, Molecular dynamics with coupling to an external bath, J. Chem. Phys. 81 (1984) $3684-3690$.

[23] B. Hess, H. Bekker, H.J.C. Berendsen, J.G.E.M. Fraaije, LINCS: a linear constraint solver for molecular simulations, J. Comp. Chem. 18 (1997) 1463-1472.
[24] T. Darden. D. York, L. Pedersen. Particle mesh Ewald-an $N \cdot \log (N)$ method for Ewald sums in large systems. J. Chem. Phys. 98 (1993) 10089-10092.

[25] G.N. Parkinson, M.P.H. Lee, S. Neidle, Crystal structure of parallel quadruplexes from human telomeric DNA. Nature 417 (2002) 876-880.

[26] A.M. Ferrenberg, R.H. Swendsen, Optimized Monte Carlo data analysis, Phys. Rev. Lett. 63 (1989) 1195-1198.

[27] C.W. Gardiner, Handbook of Stochastic Methods, Springer, 1990.

28] T. Mashimo, H. Sugiyama, Folding pathways of human telomeric hybrid Gquadruplex structure, Nucleic Acids Symp. Ser. 51 (2007) 239-240.

[29] K.C. Neuman, A. Nagy. Single-molecule force spectroscopy: optical tweezers, magnetic tweezers and atomic force microscopy, Nat. Methods 5 (2008) 491 505. 